\title{
An Autopsy Case of An Acute Exacerbation of Idiopathic Pulmonary Fibrosis Triggered by the Inhalation of a Waterproofing Spray
}

\author{
Takako Kawaguchi ${ }^{1}$, Takashi Tachiwada ${ }^{1}$, Kei Yamasaki ${ }^{1}$, Kei Nakamura ${ }^{1}$, Eisuke Katafuchi ${ }^{2}$, \\ Masahiro Tahara ${ }^{1}$, Yu Isoshima ${ }^{1}$, Hidenori Ohira ${ }^{1}$, Hiroki Kawabata ${ }^{1}$, \\ Kanako Hara ${ }^{1}$ and Kazuhiro Yatera ${ }^{1}$
}

\begin{abstract}
:
An 82-year-old Japanese man with idiopathic pulmonary fibrosis (IPF) experienced dyspnea after using a waterproofing spray in a closed room. He presented with hypoxemia and his chest computed tomography showed additive bilateral diffuse ground-glass attenuation on fibrosis, which was diagnostic of an acute exacerbation of IPF (AE-IPF). Combined treatment with high-dose corticosteroids and immunosuppressants were ineffective, and he later died of respiratory failure. Autopsy findings showed diffuse alveolar damage with honeycombing. His medical history and autopsy histopathology suggested AE-IPF caused by the inhalation of a waterproofing spray.
\end{abstract}

Key words: autopsy, acute exacerbation, diffuse alveolar damage, idiopathic pulmonary fibrosis, interstitial lung disease, waterproofing spray

(Intern Med Advance Publication)

(DOI: 10.2169/internalmedicine.8330-21)

\section{Introduction}

Waterproofing sprays are commercially available and generally used on clothing and leather products worldwide for their water-repellent properties. Waterproofing spray is generally composed of volatile solvents (such as isopropanol or xylene), water-repellent agents (such as a fluorinated resin), and spray agents (such as butane or compressed air) (1). The inhalation of the fluorinated compound resins used as a water repellent is known to cause acute lung injury (ALI) (2).

Waterproofing sprays used in a closed room often cause respiratory symptoms such as coughing and dyspnea, even when used for only a short duration (1-3). Pulmonary impairment caused by the inhalation of waterproofing sprays usually shows a spontaneous improvement (4), or responds to corticosteroid treatment even in patients with severe or prolonged lung injury $(3,5,6)$. In patients with preexisting lung diseases such as emphysema, prolonged and/or severe lung damage caused by waterproofing sprays have been reported $(7,8)$, however, fatal cases are rare.

We herein report the first autopsy case of a fatal acute exacerbation of idiopathic pulmonary fibrosis (AE-IPF) caused by the inhalation of a waterproofing spray.

\section{Case Report}

Following a physical examination in August 2017, an 82year-old Japanese man was referred to a hospital under a suspicion of interstitial lung disease (ILD). He had no history of medications, smoking, or exposure to hazardous materials, and no physical findings suggestive of any collagen vascular diseases. High-resolution computed tomography (HRCT) of his chest revealed bilateral peripheral interstitial reticular opacities, cystic lesions, and traction bronchiectasis

${ }^{1}$ Department of Respiratory Medicine, University of Occupational and Environmental Health, Japan and ${ }^{2}$ Department of Pathology, University of Occupational and Environmental Health, Japan 


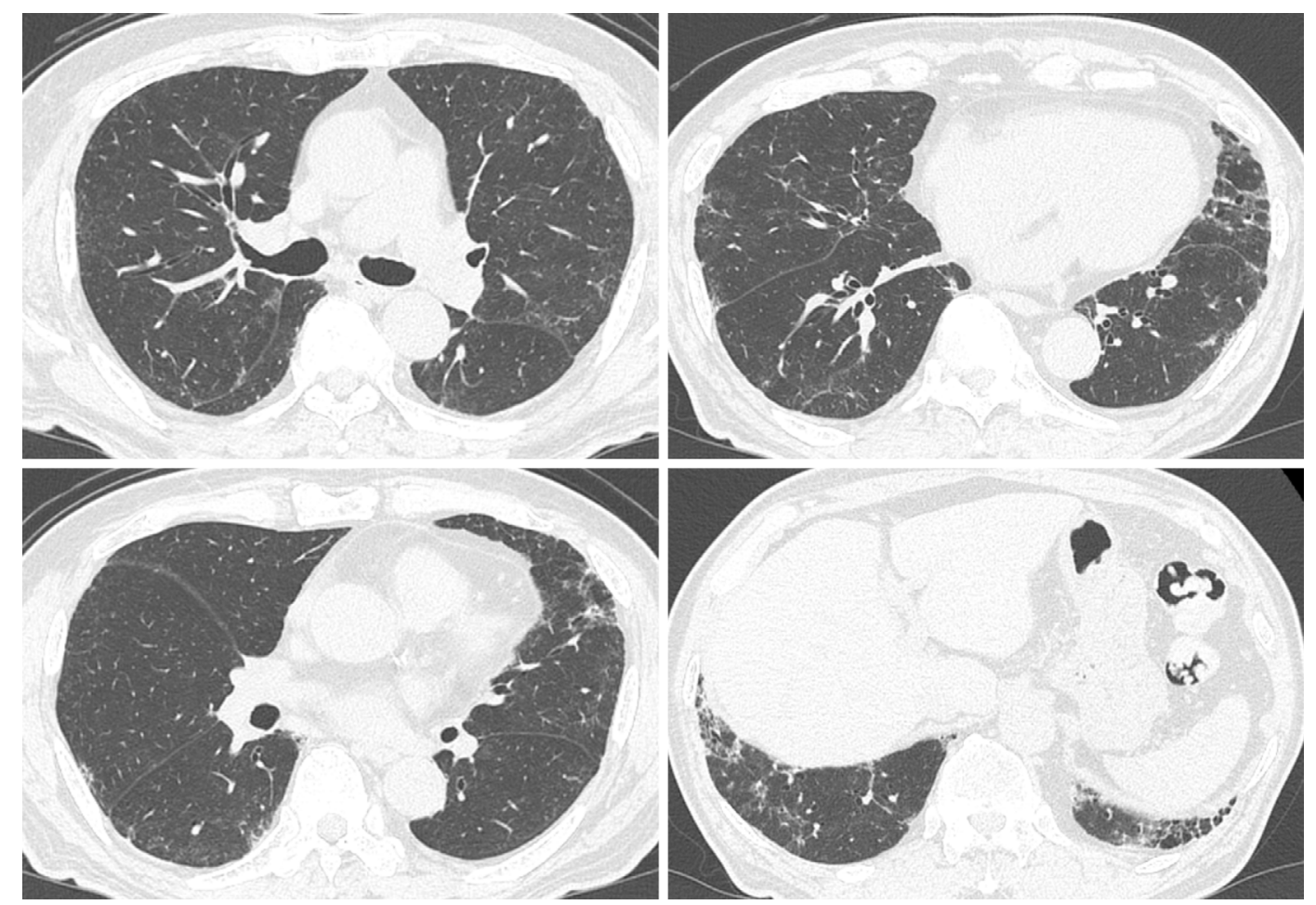

Figure 1. The HRCT scan findings of the chest at the time of IPF diagnosis in 2017 showed interstitial reticular opacities, cystic lesions, and traction bronchiectasis in the bilateral lower lobes with a pleural predominance.

in the bilateral lower lobes (Fig. 1). According to the IPF official guidelines in 2011 (10), his HRCT pattern was considered to be suggestive of "possible usual interstitial pneumonia (UIP)," and he was thus diagnosed with ILD. He had been asymptomatic and his chest HRCT findings demonstrated a very slow progression; thus, he received no treatments for ILD. In September 2019, he experienced dyspnea at rest immediately after using a can $(360 \mathrm{~mL})$ of waterproofing spray on six pairs of shoes in a closed room, and he immediately visited our hospital. This was the first time he had used waterproofing spray. He had hypoxemia $\left(\mathrm{SpO}_{2}\right.$ $88 \%$ ) on admission two hours after inhalation exposure to waterproofing spray gas, and his chest HRCT showed bilateral additive ground-glass attenuation (GGA) on the reticular opacities with traction bronchiectasis (Fig. 2A). Prior to admission, he had no history of inhalation exposure to hazardous materials except for waterproofing spray. His laboratory findings showed increased C-reactive protein (CRP), 10.07 $\mathrm{mg} / \mathrm{dL}$; lactate dehydrogenase (LDH), 265 IU/L; and surfactant protein-D (SP-D), $437 \mathrm{ng} / \mathrm{mL}$. His autoantibodies and fungal infection markers, such as beta-D glucan, were all negative (Table). Bronchoalveolar lavage (BAL) was performed from the right middle lobe, and the bronchoalveolar lavage fluid (BALF) specimen revealed a total cell count of $0.9 \times 10^{5} / \mathrm{mL}$ with macrophages (54\%), neutrophils (33\%), lymphocytes (10\%), and eosinophils (3\%). There were no infectious pathogens, such as bacteria or fungi, and malignant cells. Electrocardiography and echocardiography findings showed no evidence of heart impairments. Tests for
SARS-CoV-2 such as polymerase chain reaction were not performed because he was admitted our hospital before the SARS-CoV-2 pandemic. In addition, other causes of AE-IPF such as drugs, infection, and cardiogenic pulmonary edema were excluded; he was diagnosed with acute respiratory failure due to AE-IPF triggered by the inhalation of a waterproofing spray. In accordance with the AE-IPF treatment protocol, high-dose intravenous corticosteroids (methylprednisolone $1 \mathrm{~g}$ /day for 3 days) was initiated on the first day of treatment followed by prednisolone $60 \mathrm{mg} /$ day $(1 \mathrm{mg} / \mathrm{kg} /$ day). His $\mathrm{SpO}_{2}$ improved to $96 \%\left(\mathrm{O}_{2}, 4 \mathrm{~L} / \mathrm{min}\right.$; nasal cannula) on day 3 after starting the corticosteroid treatment, but the symptoms gradually worsened on day 4, which prompted the addition of cyclosporin A to the corticosteroids on the same day. His $\mathrm{SpO}_{2}$ continuously worsened to 93\% (15 L/min, reservoir mask) on day 9 with an exacerbation of GGA with honeycombing on his chest HRCT (Fig. 2B). Therefore, methylprednisolone $1 \mathrm{~g} /$ day for 3 days was re-administered from day 9 to day 11 . However, his respiratory condition continued to worsen, and he eventually died of respiratory failure on day 17 (Fig. 3).

An autopsy was performed 3 hours after his death, and both lungs were poorly inflated and their weight had increased. The histopathological findings of his lungs showed bilateral diffuse hyaline membrane formation and organizing changes, compatible with diffuse alveolar damage (DAD) in the exudative phase to organizing phase of AE-IPF (Fig. 4A). Pathologically, dorsal lesions of bilateral lower lobes also showed cystic dilatated air spaces with fibrosis 


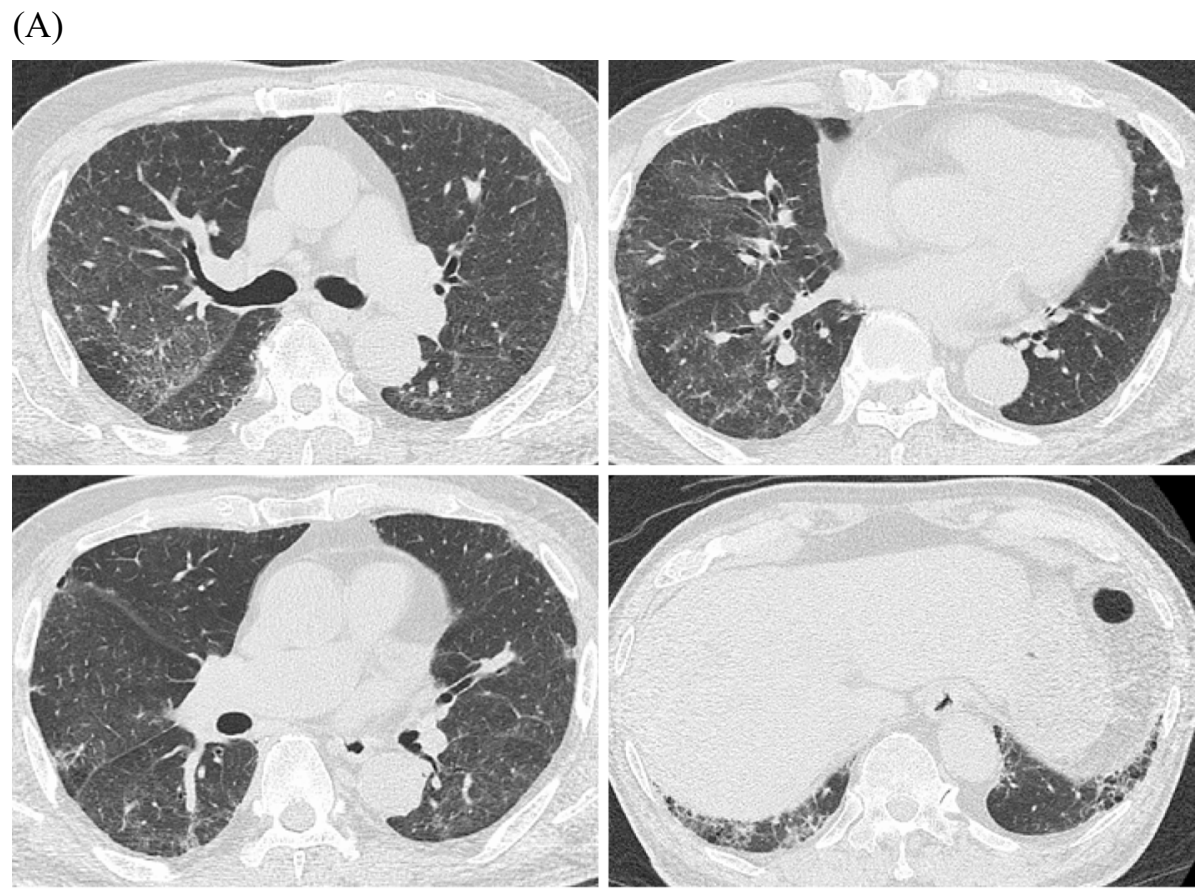

(B)

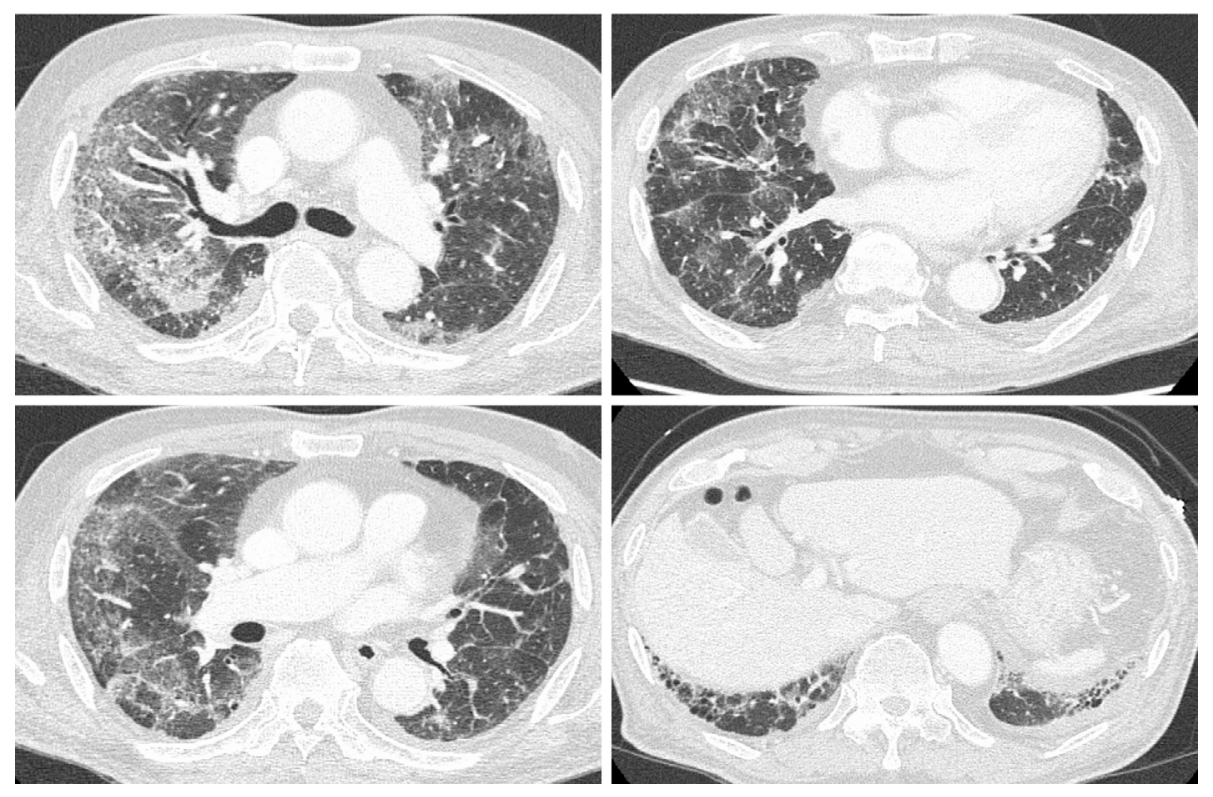

Figure 2. (A) The HRCT scans of the chest on admission showed diffuse GGA bilaterally, with reticular opacities with traction bronchiectasis in the bilateral lower lobes of the lung with a predominance of subpleural areas. (B) Follow-up CT after 9 days of hospitalization showed bilateral diffuse GGA with an exacerbation of traction bronchiectasis and mixed consolidation.

and honeycombing (Fig. 4B). Granuloma or cross-linkage formation (possibly indicative of chronic hypersensitivity pneumonitis), lymphoid follicles (possibly indicative of collagen vascular diseases), and eosinophilic infiltrates (possibly indicative of eosinophilic pneumonia) were not observed, and he was pathologically diagnosed to have UIP/ IPF. Although the autopsy findings were not informative of the cause of his AE-IPF, the inhalation of a waterproofing spray used just before the initiation of his acute respiratory symptoms which led to fatal respiratory failure was the most likely cause of AE-IPF.

\section{Discussion}

We herein present the first autopsy case of a patient who died of AE-IPF caused by the inhalation of a waterproofing spray. To the best of our knowledge, this is the first autopsy case report regarding fatal AE-IPF triggered by the inhalation of a waterproofing spray.

We diagnosed the present case as AE-IPF due to inhala- 
Table. Results of Peripheral Blood Analysis on Admission and BALF Findings ${ }^{1}$.

\begin{tabular}{|c|c|c|c|c|c|}
\hline \multicolumn{2}{|l|}{ Blood cell counts } & \multicolumn{2}{|c|}{ Blood chemistry } & \multicolumn{2}{|l|}{ Serology } \\
\hline WBC & $9,200 / \mu \mathrm{L}$ & $\mathrm{TP}$ & $5.9 \mathrm{~g} / \mathrm{dL}$ & CRP & $10.07 \mathrm{mg} / \mathrm{dL}$ \\
\hline Neutrophils & $94.9 \%$ & Alb & $3.0 \mathrm{~g} / \mathrm{dL}$ & KL-6 & $292 \mathrm{U} / \mathrm{mL}$ \\
\hline Lymphocytes & $3.9 \%$ & T-bil & $1.2 \mathrm{mg} / \mathrm{dL}$ & SP-D & $437 \mathrm{ng} / \mathrm{mL}$ \\
\hline Eosinophils & $0.0 \%$ & AST & 46 IU/L & Rheumatoid factor & $<0.5 \mathrm{U} / \mathrm{mL}$ \\
\hline Monocytes & $1.1 \%$ & ALT & $26 \mathrm{IU} / \mathrm{L}$ & ANA & $<40$ titer \\
\hline Basophils & $0.1 \%$ & LDH & $265 \mathrm{IU} / \mathrm{L}$ & $\beta$-D glucan & $<6.0 \mathrm{pg} / \mathrm{mL}$ \\
\hline $\mathrm{RBC}$ & $401 \times 10^{4} / \mu \mathrm{L}$ & ALP & $214 \mathrm{IU} / \mathrm{L}$ & \multicolumn{2}{|c|}{ BALF Findings (rt. $\mathrm{B}^{4 \mathrm{~b}}$ ) } \\
\hline $\mathrm{Hb}$ & $13.1 \mathrm{~g} / \mathrm{dL}$ & $\gamma$-GTP & $23 \mathrm{IU} / \mathrm{L}$ & Recovery & $92 / 150 \mathrm{~mL}$ \\
\hline $\mathrm{Ht}$ & $36.3 \%$ & BUN & $16 \mathrm{mg} / \mathrm{dL}$ & Total cell count & $0.9 \times 10^{5} / \mathrm{mL}$ \\
\hline Platelets & $12.1 \times 10^{4} / \mu \mathrm{L}$ & Cre & $0.66 \mathrm{mg} / \mathrm{dL}$ & Macrophages & $54 \%$ \\
\hline Coagulation & & CK & $65 \mathrm{IU} / \mathrm{L}$ & Neutrophils & $33 \%$ \\
\hline PT & $12.2 \mathrm{~s}$ & $\mathrm{Na}$ & $141 \mathrm{mmol} / \mathrm{L}$ & Lymphocytes & $10 \%$ \\
\hline PT-\% & $86.4 \%$ & K & $3.8 \mathrm{mmol} / \mathrm{L}$ & Eosinophils & $3 \%$ \\
\hline PT-INR & $1.06 \mathrm{~s}$ & $\mathrm{Cl}$ & $106 \mathrm{mmol} / \mathrm{L}$ & $\mathrm{CD} 4 / 8$ ratio & 2.5 \\
\hline APTT & $28.0 \mathrm{~s}$ & & & \multicolumn{2}{|c|}{ Arterial blood gas $\left(\mathrm{O}_{2} 4 \mathrm{~L} / \mathrm{min}\right)$} \\
\hline FDP & $4.7 \mu \mathrm{g} / \mathrm{mL}$ & & & $\mathrm{pH}$ & 7.461 \\
\hline \multirow[t]{3}{*}{ D-dimer } & $1.7 \mu \mathrm{g} / \mathrm{mL}$ & & & $\mathrm{PaCO}_{2}$ & $34.9 \mathrm{mmHg}$ \\
\hline & & & & $\mathrm{PaO}_{2}$ & $79 \mathrm{mmHg}$ \\
\hline & & & & $\mathrm{HCO}_{3}^{-}$ & $24.3 \mathrm{mmol} / \mathrm{L}$ \\
\hline
\end{tabular}

${ }^{1}$ WBC: white blood cell, RBC: red blood cell, Hb: hemoglobin, Ht: hematocrit, FDP: fibrin/fibrinogen degradation products, TP: total protein, Alb: albumin, T-bil: total bilirubin, AST: aspartate aminotransferase, ALT: alanine aminotransferase, LDH: lactate dehydrogenase, ALP: alkaline phosphatase, $\gamma$-GTP: gamma-glutamyl transferase, BUN: blood urea nitrogen, Cre: creatinine, CK: creatine kinase, CRP: C-reactive protein, KL-6: sialylated carbohydrate antigen-6, SP-D: pulmonary Surfactant Protein-D, ANA: antinuclear antibody, BALF: Bronchoalveolar lavage fluid
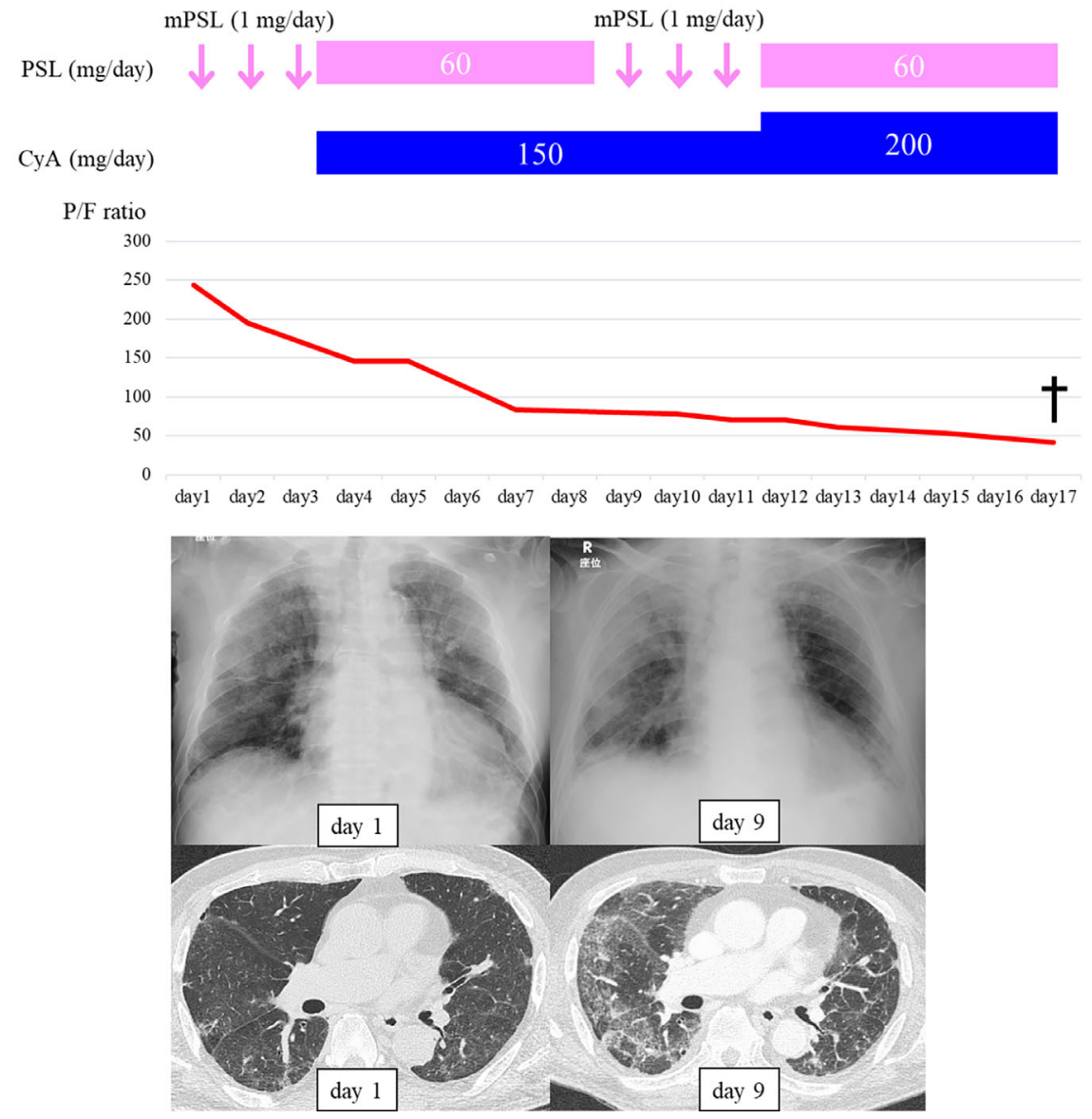

Figure 3. Clinical course of the patient. CyA: cyclosporin A, mPSL: methylprednisolone, P/F: $\mathrm{PaO}_{2} / \mathrm{FiO}_{2}$, PSL: prednisolone 
(A)

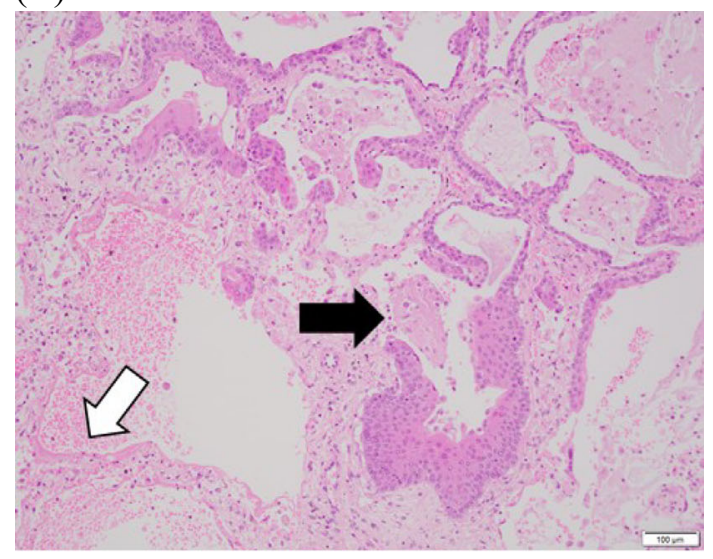

(B)

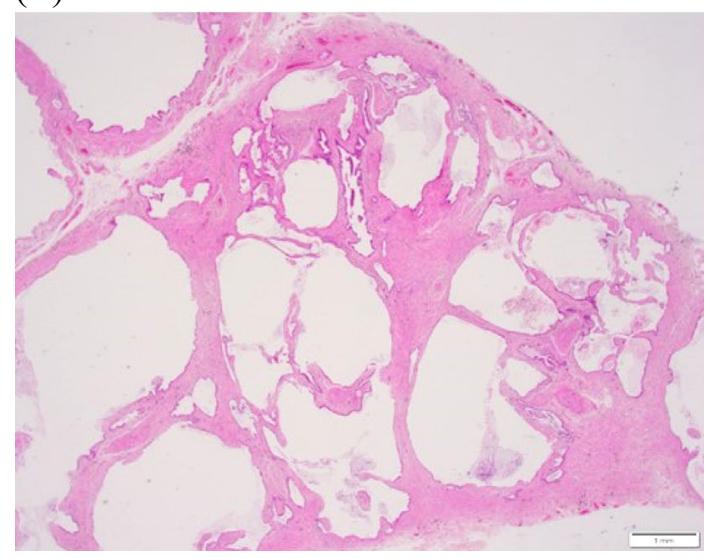

Figure 4. (A) The autopsy lung tissue showed pathological findings suggestive of DAD in the advancement and organizing phases, including organizing lesions the alveolar space (black arrow) and vitreous membrane formation (white arrow). (B) The autopsy lung tissue showed cystic dilatation and honeycombing predominantly in the lower lobes, thus suggesting that the patient had chronic lung fibrosis.

tion of a waterproofing spray based on the following reasons: Our patient presented with dyspnea two hours after inhaling the waterproofing spray. According to the reported data of the onset of symptoms due to inhalation of waterproofing spray in the Japanese Poisoning Center, approximately one-third of the cases showed dyspnea within one hour, and the remaining two-thirds showed dyspnea within approximately half a day (11). Similar to the findings of previous reports $(5,12)$, a relative central distribution of bilateral GGA mainly in the upper lobes sparing the subpleural areas were observed on chest HRCT which were obtained soon after inhaling the waterproofing spray in our patient.

The inhalation of a waterproofing spray, especially a fluorinated compound resin used as a water repellent, causes ALI (2). In addition to the inhalation of a waterproofing spray, acute eosinophilic pneumonitis (AEP) and an exacerbation of chronic hypersensitivity pneumonitis (CHP) should also be considered in the differential diagnosis of early respiratory disorder following antigen inhalation (13). However, it is difficult to differentiate between these diseases based on the clinical findings. The antigen inhalant associated with AEP and CHP is smoke and a repetitive specific antigen, respectively. Moreover, elevated eosinophils and lymphocytes in BALF are observed in patients with AEP and CHP, respectively (13). The BALF in ALI due to the inhalation of waterproofing spray are known to dominantly contain macrophages (13), but elevated neutrophils in the early phase after the inhalation of a waterproofing spray have also been reported (14). Therefore, the BALF in our patient was suggestive of the early findings of ALI triggered by the inhalation of a waterproofing spray.

The autopsy of our patient revealed weight gained by both lungs with bilateral diffuse hyaline membrane formation, organogenesis, and squamous epithelialization, consistent with the chest HRCT images of a DAD pattern in the exudative to organogenesis stages. The histopathological findings in our patient also showed cystic dilatation of the air spaces with fibrosis on the dorsal side of the bilateral lower lobes (honeycombing) thus suggesting the presence of a UIP pattern in the background (15). In addition to IPF, other ILD with UIP patterns, such as CHP and collagen vascular disease-associated ILD, should also be considered in the differential diagnosis (15). No granuloma formation, cross-linkage formation, or lymphatic follicles were observed in the lungs. As a result, the pulmonary pathological diagnosis of our patient was UIP/IPF. Moreover, his HRCT at the time of his first visit in 2017 showed a "possible UIP" pattern according to the IPF official guidelines in 2011 (10), similar to the "probable UIP" pattern stated in the IPF official guidelines of 2018 (15); in combination with his pathological findings and radiological findings, he was diagnosed with a "UIP" pattern based on the IPF official guidelines of 2018 (15). Few reports of murine pulmonary pathology of lung injury caused by inhalation of waterproofing spray revealed that fluoropolymers were the main causative ingredients for alveolar damage; alveolar collapse, thickening of alveolar septa, inflammatory hyperemia, and leaky hemorrhaging were observed an hour after inhalation exposure (16). The histopathological findings in our patient were not consistent with the findings of murine lung injury caused by inhalation of waterproofing spray. Instead, the pulmonary pathological findings in our patient were compatible with typical the pathological findings of AE-IPF with the presence of dense fibrotic lesions in the background (UIP pattern) and additive vitreous membrane formation (DAD pattern) (17).

ALI caused by the inhalation of waterproofing sprays often resolves spontaneously (4) or by corticosteroid treatment $(3,5,6)$. A few cases require ventilator (18); it was only required in one fatal but suicidal case with chemical parasympathetic nerve stimulation and subsequent cardiac arrest due to vasovagal reflex in addition to respiratory failure (19). Our patient was diagnosed with AE-IPF with severe respiratory failure after the inhalation of waterproofing spray. Despite performing aggressive treatment with highdose corticosteroids and immunosuppressants, no improve- 
ment in the patient symptoms was achieved, thus resulting in a fatal outcome.

\section{Conclusions}

We herein presented the first autopsy case of a patient with fatal AE-IPF triggered by a waterproofing spray inhalation. Lung injury due to the inhalation of waterproofing sprays has been reported, and it should be generally considered that inhaling a waterproofing spray can lead to severe or even fatal respiratory failure. Individuals, especially patients with lung diseases should be advised to use gaseous products including waterproofing sprays in well-ventilated areas.

The authors state that they have no Conflict of Interest (COI).

\section{References}

1. Daubert GP, Spiller H, Crouch BI, et al. Pulmonary toxicity following exposure to waterproofing grout sealer. J Med Toxicol 5: 125-129, 2009.

2. Vernez D, Bruzzi R, Kupferschmidt H, et al. Acute respiratory syndrome after inhalation of waterproofing sprays: a posteriori exposure-response assessment in 102 cases. J Occup Environ Hyg 3: 250-261, 2006.

3. Harada T, Hirabayashi Y, Takayama-Isagawa Y, et al. Pulmonary Injury from Waterproofing Spray During a Hike. Wilderness Environ Med 28: 327-331, 2017.

4. Kikuchi R, Itoh M, Uruma T, et al. Diffuse alveolar hemorrhage after use of a fluoropolymer-based waterproofing spray. Springerplus 4: 270, 2015.

5. Wallace GM, Brown PH. Horse rug lung: toxic pneumonitis due to fluorocarbon inhalation. Occup Environ Med 62: 414-416, 2005.

6. Nakazawa A, Hagiwara E, Harada S, et al. Surgically proven desquamative interstitial pneumonia induced by waterproofing spray. Intern Med 53: 2107-2110, 2014.

7. Hashimoto K, Arita K, Kajihara T, et al. Two cases of lung injury due to inhalation of waterproofing spray--with special reference to pulmonary function disorder. Nihon Kokyuki Gakkai Zasshi 47:
367-371, 2009 (in Japanese, Abstract in English).

8. Kondo A, Yanagawa Y, Omori K, et al. Importance of smoking and the occurrence of acute poisoning due to waterproof spray. Acute Med Surg 1: 191-194, 2014.

9. Collard HR, Ryerson CJ, Corte TJ, et al. Acute Exacerbation of Idiopathic Pulmonary Fibrosis. An International Working Group Report. Am J Respir Crit Care Med 194: 265-275, 2016.

10. Raghu G, Collard HR, Egan JJ, et al. An official ATS/ERS/JRS/ ALAT statement: idiopathic pulmonary fibrosis: evidence-based guidelines for diagnosis and management. Am J Respir Crit Care Med 183: 788-824, 2011.

11. Hatano Y, Imabeppu F, Nomura N. News from the Japan Poison Information Center: Respiratory hazard due to inhalation of commercial waterproofing sprays. The Japanese Journal of Clinical Toxicology 23: 73-78, 2010 (in Japanese, Abstract in English).

12. Heinzer R, Ribordy V, Kuzoe B, et al. Recurrence of acute respiratory failure following use of waterproofing sprays. Thorax 59: 541-542, 2004.

13. Shimoda M, Tanaka Y, Fujiwara K, et al. Waterproofing sprayassociated pneumonitis review. Medinica 100: e25054, 2021.

14. Shimizu K, Shiba Y, Tanoue M, et al. A case of protracted lung injury caused by inhalation of waterproofing spray. Journal of the Japan Society for Respiratory Endoscopy 36: 78-82, 2014.

15. Raghu G, Remy-Jardin M, Myers JL, et al. Diagnosis of Idiopathic Pulmonary Fibrosis. An Official ATS/ERS/JRS/ALAT Clinical Practice Guideline. Am J Respir Crit Care Med 198: e44e68, 2018.

16. Yamashita M, Tanaka J. Pulmonary collapse and pneumonia due to inhalation of a waterproofing aerosol in female CD-1 mice. $\mathrm{J}$ Toxicol Clin Toxicol 33: 631-637, 1995.

17. Collard HR, Moore BB, Flaherty KR, et al. Acute exacerbations of idiopathic pulmonary fibrosis. Am J Respir Crit Care Med 176: 636-643, 2007.

18. Hitosugi M, Maruyama K, Kitamura O, et al. Suicial death caused by spraying of aerosol water-epellent spray. The Japanese Journal of Clinical Toxicology 10: 289-292, 1997.

19. Sawamoto T, Morita S, Watanabe Y, et al. A Case of Acute Respiratory Distress Syndrome Caused by Waterproofing Spray Inhalation. Tokai J Exp Clin Med 43: 106-110, 2018.

The Internal Medicine is an Open Access journal distributed under the Creative Commons Attribution-NonCommercial-NoDerivatives 4.0 International License. To view the details of this license, please visit (https://creativecommons.org/licenses/ by-nc-nd/4.0/).

(C) The Japanese Society of Internal Medicine Intern Med Advance Publication 